\title{
Case report and literature review: transient Inab phenotype and an agglutinating anti-IFC in a patient with a gastrointestinal problem
}

\author{
Mark H. Yazer, W. John Judd, Robertson D. Davenport, Louann R. Dake, Christine Lomas-Francis, \\ Kim Hue-Roye, Vivien Powell, and Marion Reid
}

BACKGROUND: The Inab phenotype is a rare deficiency of all Cromer antigens. These antigens are carried on the decay-accelerating factor (DAF, CD55) molecule that is attached to the red blood cell (RBC) membrane by a glycosylphosphatidylinositol (GPI) anchor. Although typically inherited, an acquired and transient form of the Inab phenotype also exists. A patient with the triad of transient Inab phenotype, a direct-agglutinating anti-IFC, and gastrointestinal (Gl) abnormalities is reported.

CASE REPORT: An 18-month-old boy with

gastroesophageal reflux disease requiring a feeding tube, milk and soy intolerance, and severe growth retardation, as well as vision and hearing deficits from cytomegalovirus infection, was identified when pretransfusion testing revealed a potent panagglutinin (titer $>2000$ at $4^{\circ} \mathrm{C}$ ). This antibody did not react with $\operatorname{Dr}(\mathrm{a}-)$ and IFC RBCs, and the autocontrol was negative. The patient's RBCs lacked CD55 by flow cytometric techniques but had normal levels of CD59 and antigens such as $\mathrm{Yt}^{\mathrm{a}}$ and Emm, carried on GPI-linked proteins, thus excluding paroxysmal nocturnal hemoglobinuria. Several months after initial detection, the anti-IFC was virtually undetectable and his cells reacted weakly with anti-IFC, anti-Dra , and anti-CD55. RBCs from the propositus' parents and brother demonstrated normal CD55 and CD59 expression.

CONCLUSION: This is the first example of a directagglutinating anti-IFC. The cause of the transient depression in CD55 protein (and thus Cromer system antigens) and appearance of anti-IFC remains unknown, as does the relationship between the patient's GI system abnormalities and these serologic findings.
$\Gamma$ here are 14 antigens in the Cromer blood group system, 11 of high incidence and three of low incidence (Table 1). ${ }^{1-6}$ All of these antigens are located on the complement regulating protein decay-accelerating factor (DAF, CD55), which is tethered to the red blood cell (RBC) membrane by a glycosylphosphatidylinositol (GPI) anchor. ${ }^{7}$ There are three molecular backgrounds that give rise to weakened or absent expression of Cromer antigens. First, somatic mutations in the X-linked phosphatidylinositol-glycan-complementation class A $(P I G-A)$ gene result in a biosynthetic defect in GPI anchor synthesis and hence the absence of all GPI-linked proteins on the RBC surface. ${ }^{8}$ The absence or reduction of these proteins on RBCs occurs in paroxysmal nocturnal hemoglobinuria $(\mathrm{PNH})$. In its most extreme form, when the two main regulators of complement, CD55 and membrane inhibitor of reactive lysis (CD59) are completely lacking on the RBC surface; intravascular hemolysis occurs and has been associated with thrombosis (reviewed in Rosse and Nishimura ${ }^{9}$ ).

Second, reduced Cromer system antigen expression occurs in the case of the rare $\operatorname{Dr}(\mathrm{a}-)$ RBCs. ${ }^{10}$ The molecular basis for this phenotype is a single-nucleotide polymor-

ABBREVIATIONS: DAF = decay-accelerating factor; GPI = glycosylphosphatidylinositol; SNP = single-nucleotide polymorphism.

From the Institute for Transfusion Medicine and Department of Pathology, University of Pittsburgh, Pittsburgh, Pennsylvania; the Department of Pathology, University of Michigan, Ann Arbor, Michigan; and the New York Blood Center, New York, New York. Address reprint requests to: Mark Yazer, MD, 3636 Boulevard of the Allies, Pittsburgh, PA 15213; e-mail: myazer@itxm.org. Funded in part by an NIH Specialized Center of Research (SCOR) grant in transfusion medicine and biology (HL54449).

Received for publication November 7, 2005; revision received February 16, 2006, and accepted February 16, 2006.

doi: 10.1111/j.1537-2995.2006.00933.x

TRANSFUSION 2006;46:1537-1542. 
phism (SNP) in exon 5 of $D A F, 596 \mathrm{C}>\mathrm{T}$, which results in alternative splicing of the $D A F$ mRNA such that the resulting major transcript lacks 44 nucleotides. ${ }^{10-12}$ In addition, this alternative splicing shifts the reading frame and introduces a premature stop codon; the translated protein cannot attach to the membrane and is not present on the membrane of the RBCs. ${ }^{12} \mathrm{~A}$ small minority of the $D A F$ mRNA transcripts, however, contain this SNP but do not utilize the cryptic splice acceptor site. ${ }^{13}$ These minor transcripts are translated normally with the result that there is a reduced copy number of CD55 and weakened expression of Cromer system antigens on $\operatorname{Dr}(\mathrm{a}-)$ cells. ${ }^{13}$

Third, Inab phenotype RBCs, named after the original Japanese propositus, lack all Cromer system antigens. ${ }^{14}$ This rare phenotype has been previously reported in nine people and is typically recognized by the presence of an alloantibody (anti-IFC) in their serum to a high-incidence antigen of the Cromer system absent on these cells (Table 2) ${ }^{14-20}$ Several SNPs have been identified underlying this phenotype. The original Japanese propositus ${ }^{14}$ and another unrelated Japanese individual ${ }^{18}$ demonstrated a nonsense $261 \mathrm{G}>\mathrm{A}$ polymorphism in exon 2 of the $D A F$ gene rendering the protein unable to be tethered to the
GPI anchor. Two Japanese individuals with the Inab phenotype were reported to have a different SNP, 263C $>$ A, which causes a 26-bp deletion in the DAF mRNA secondary to the activation of a cryptic splice site. ${ }^{17,19}$ Recently, another Japanese proband with the Inab phenotype and a novel nonsense SNP (508C $>$ T, Arg136Stop) was identified. ${ }^{20}$ The molecular basis for the Inab phenotype in several patients of Jewish, ${ }^{15}$ Italian, ${ }^{16}$ and African American ${ }^{18}$ descent has not been elucidated. Curiously, some of the first patients with the Inab phenotype were noted to have a variety of gastrointestinal (GI) system abnormalities, whereas others with the Inab phenotype lacked these problems (Table 2).

In addition, there are two reports of transient IFCnegative individuals. One was a previously healthy Kenyan national with $\mathrm{HbS}$ trait, unexplained splenic infarcts, and transient Inab phenotype. ${ }^{21}$ The patient had no GI abnormalities and had never received a transfusion, yet an antiIFC-reactive at indirect antiglobulin test (IAT) was present in his serum sample, and his cells were clearly lacking CD55 by flow cytometric analysis. ${ }^{21}$ More recently, an elderly female patient with chronic lymphocytic leukemia and transient Inab phenotype with anti-IFC was reported..$^{22}$ We report another example of a patient with transient Inab phenotype, who unlike the previous transient Inab phenotype propositi, had GI abnormalities and a direct agglutinating anti-IFC.

\section{CASE REPORT}

The propositus is a Caucasian male born at 38 weeks' gestation with congenital cytomegalovirus disease causing blindness and deafness. The patient had severe growth retardation (height, first percentile; weight, 0 percentile) and suffered significant gastroesophageal reflux disease as well as milk and soy

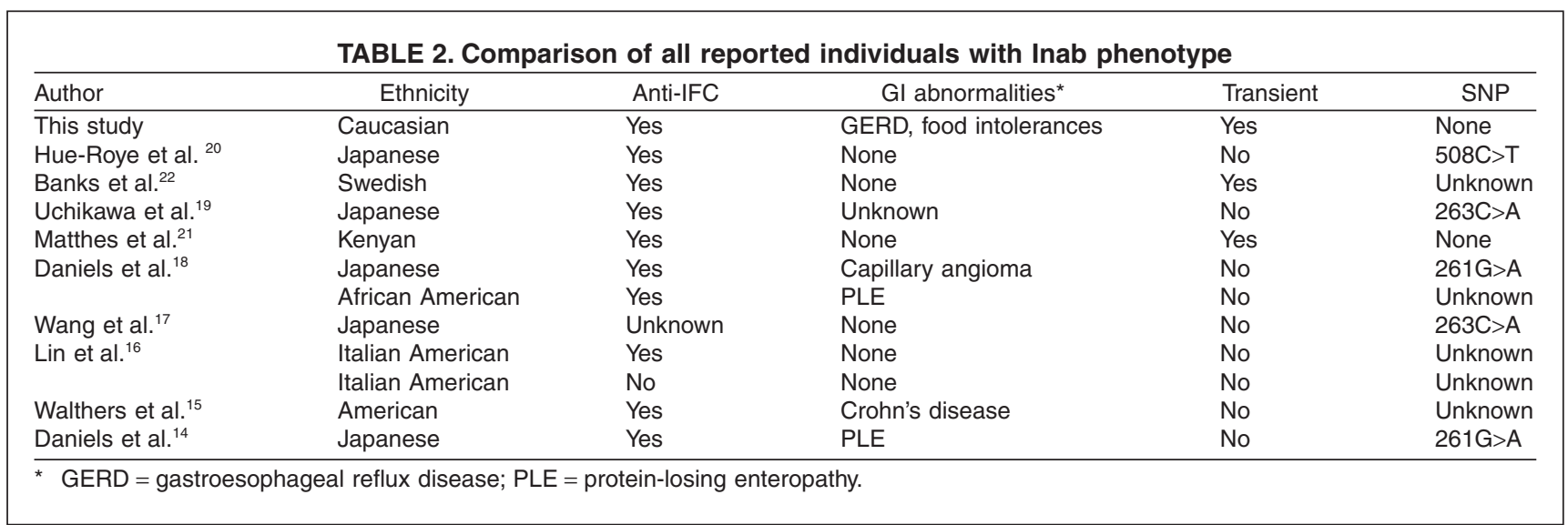


intolerances such that a feeding tube was required. At age 1 year (April 2004), he came to the attention of the blood bank when routine pretransfusion testing, performed in advance of cochlear implant surgery, revealed an $\mathrm{ABO}$ typing discrepancy due to a potent panagglutinin. Additional samples were obtained when the patient returned for a follow-up visit at age 16 months (August 2004). At that time his complete blood count was within normal limits. Additional samples were also obtained after surgery at age 18 months (October 2004).

\section{MATERIALS AND METHODS}

\section{RBC serology}

Standard serologic techniques were performed throughout this case study investigation. ${ }^{23}$ Reagent RBCs and antibodies to blood group antigens were from either ImmucorGamma (Norcross, GA) or Ortho Clinical Diagnostics (Raritan, NJ), or from the private collections of our institutions. Gel column technology was from Ortho Clinical Diagnostics.

Immunoglobulin G (IgG) monoclonal antibodies to CD55 and CD59 were a gift from G. Halverson of the Lindsay F. Kimball Research Institute at the New York Blood Center and were tested against RBCs in gel columns containing mouse anti-IgG (MicroTyping Systems, Pompano Beach, FL). Escherichia coli (E. coli 12F), containing the 075X adhesin (Dra adhesin), ${ }^{24}$ was a gift from C.F. Marrs (School of Public Health, University of Michigan). Bacterial hemagglutination tests were performed on buffered gel cards (Ortho Clinical Diagnostics) as described previously. ${ }^{25}$

\section{Flow cytometry}

Washed RBCs (50 $\mu \mathrm{L}, 3 \%$ suspension) were incubated with IgG monoclonal anti-CD55 and CD59 $(50 \mu \mathrm{L})$ for 30 minutes at room temperature. After being washed, the RBCs were incubated with fluorescein isothiocyanate (FITC)-labeled mouse anti-IgG, washed, and then resuspended in $500 \mu \mathrm{L}$ of phosphate-buffered saline-1 percent paraformaldehyde. After fixation, cells were analyzed by one-color flow cytometry (FACScan, Becton Dickinson, Hialeah, FL) with the accompanying software (Cell-Quest, Becton Dickinson). Cells were gated on forward and orthogonal scatter, and the percentage FITC-positive cells was determined. All samples were stained in duplicate and recorded as the mean percent positive cells and mean channel fluorescence.

\section{Sequencing of DNA}

Genomic DNA was isolated from whole blood (QIAamp blood kit, Qiagen, Inc., Valencia, CA). The regions of $D A F$ that include and flank exon 2 , exon 3 , exon 4 , exon 5 , and exon 6 (which encode SCRs 1-4) were individually amplified with sets of primers derived from Banks and colleagues ${ }^{4}$ and Telen and coworkers ${ }^{26}$ with modified primers DAFSCR2R (GAGTTCTAGCATGAATGAAGGAAGGG) and DAFSCR4F (GCATCTCTTGTTGGTAATGCTGG). The primers were synthesized by Life Technologies, Inc. (Gaithersburg, MD). Two microliters of DNA per reaction was amplified by $5 \mathrm{U}$ Taq DNA polymerase (HotStarTaq, Qiagen) in a $50-\mu \mathrm{L}$ reaction mixture containing $2.0 \mathrm{mmol}$ per $\mathrm{L} \mathrm{MgCl}_{2}$ (1.5 mmol/L $\mathrm{MgCl}_{2}$ for exon 2 primers), $1 \times$ PCR buffer, $0.2 \mathrm{mmol}$ per L dNTPs, and $100 \mathrm{ng}$ of forward and reverse primer. Amplification was achieved over 35 cycles with a final extension time of 10 minutes. The PCR products were sequenced directly in the Laboratory of Microchemistry at the New York Blood Center with an automated DNA sequencer (ABI 373XL, Applied Biosystems, Inc., Foster City, CA), and reagents (ABI Big Dye reagents with BD Half-Term, GenPak, Stony Brook, NY). The PCR products were sequenced in both directions.

\section{RESULTS}

\section{Serology}

April through August 2004. The following description combines the serologic findings from the propositus' April and August 2004 blood samples. Unexpected reactivity with $A_{1}$ cells on the serum $A B O$ typing of this apparent group A patient was noted. Additional studies revealed a strongly reactive panagglutinin $\left(2-3+^{S}\right)$ in immediate-spin tests with all panel cells tested, including a sample from cord blood. Much weaker (microscopic $1+$ to $1+^{s}$ ) reactivity was noted with all panel cells tested at albumin $37^{\circ} \mathrm{C}$, at albumin IAT, and by papain IAT. The autologous control was negative in all phases of testing. The antibody failed to agglutinate cells when saline was then added in place of AHG reagent in the IAT.

The maximum titer of this panagglutinin was 2000 at $4^{\circ} \mathrm{C}$. It agglutinated $\mathrm{RBCs}$ of the following rare phenotypes: Emm-, $\mathrm{Gy}(\mathrm{a}-), \mathrm{M}^{\mathrm{k}} \mathrm{M}^{\mathrm{k}}, \mathrm{p}$, and $\mathrm{P}_{2}{ }^{\mathrm{k}}$ and did not agglutinate $\operatorname{Dr}(\mathrm{a}-)$, IFC-, and type III PNH. The antigen recognized by this panagglutinin was ficin-, papain-, and trypsin-resistant but $\alpha$-chymotrypsin-sensitive. The failure of this antibody to agglutinate Dr(a-), IFC-, and type III PNH RBCs, along with its reaction pattern with enzyme-treated RBCs, was suggestive of an antibody to a high-prevalence antigen of the Cromer blood group system.

The patient's RBCs were negative in the DAT. The extended phenotype of his RBCs revealed that he was positive for the following antigens: A, D, C, E, c, e, M, S, s, Fy, $\mathrm{Fy}^{\mathrm{b}}, \mathrm{Jk}^{\mathrm{a}}, \mathrm{Jk}^{\mathrm{b}}, \mathrm{Wr}^{\mathrm{b}}, \mathrm{Yt}^{\mathrm{a}}, \mathrm{I}, \mathrm{P}, \mathrm{Vel}$, and $\mathrm{Emm}$. His RBCs were negative for the following antigens: N, P1, K, Dra , and IFC. His RBCs did not react with anti-CD55 when tested on mouse anti-IgG gel cards nor did they demonstrate agglutination in gel cards containing E. coli $12 \mathrm{~F}$ with the $075 \mathrm{X}$ 
adhesin. ${ }^{25}$ Overall, in view of the IFC-negative phenotype of the propositus, this antibody is considered to have antiIFC specificity, although typically anti-IFC would be expected to react weakly with $\operatorname{Dr}\left(\mathrm{a}^{-}\right)$RBCs.

October 2004. The antibody detecting a high-prevalence antigen of the Cromer blood group system was virtually undetectable in his serum sample; it reacted only microscopically by albumin IAT with one of six panel cells tested and also with a cord RBC sample. The propositus' plasma also reacted weakly at room temperature with four of seven RBC samples suspended in saline. As before, the patient's RBCs were negative in the DAT, but his RBC phenotype had changed such that his cells were now weakly reactive with anti-Dra and anti-IFC. His RBCs still failed to be agglutinated by E. coli $12 \mathrm{~F}$. Weak reactivity was now observed when his cells were tested with monoclonal antiCD55 by mouse anti-IgG gel cards. At this time the RBCs of his mother, father, and brother were also obtained and evaluated for the expression of CD55; these family members demonstrated normal expression of CD55.

\section{Flow cytometry}

CD55 was virtually absent from the RBCs of the propositus in August (gated events, 1.6 percent of control; Fig. 1). However, in October the number of CD55-positive cells (gated events) had increased to 28 percent of control. CD59 was expressed normally in specimens from both dates. This finding, taken together with the presence of the GPIanchored $\mathrm{Yt}^{\mathrm{a}}$ and Emm antigens on his RBCs, excludes PNH as the cause of reduced CD55 expression. The levels of CD55 and CD59 were also evaluated on RBCs from his parents and his brother in October: as in agglutination-based studies, RBCs of these family members demonstrated normal CD55 and CD59 expression.

\section{Sequencing of DNA}

Exons 2 through 6 of $D A F$ and their flanking intronic sequences were sequenced and found to be identical to the consensus sequence.

\section{DISCUSSION}

This is the first report of a patient with the triad of GI abnormalities, transient Inab phenotype, and a direct aggluti-

A control. nating antibody directed at a high-prevalence Cromer antigen. The specificity is considered to be anti-IFC based on the findings that the patient's RBCs were IFC- and that the antibody did not agglutinate IFC- or $\operatorname{Dr}(\mathrm{a}-)$ RBCs. The failure to agglutinate $\operatorname{Dr}(\mathrm{a}-)$ RBCs is most likely due to the very low levels of Cromer antigens expressed on RBCs with this phenotype. Unlike a previous case report of a patient with transient Inab phenotype with anti-IFC, ${ }^{21}$ our patient did not manifest thrombotic complications, although in both cases a generalized absence of GPI-linked proteins was excluded by the presence of normal amounts of CD59 on the RBC surface. Exons 2 through 6 of $D A F$ were consensus in both propositi. Unfortunately, we have been unable to obtain further blood samples from the current propositus and his family, nor can we be certain of the length of time the current propositus had the transient Inab phenotype with the anti-IFC. It is interesting to note that the anti-IFC reported here was a direct agglutinin that
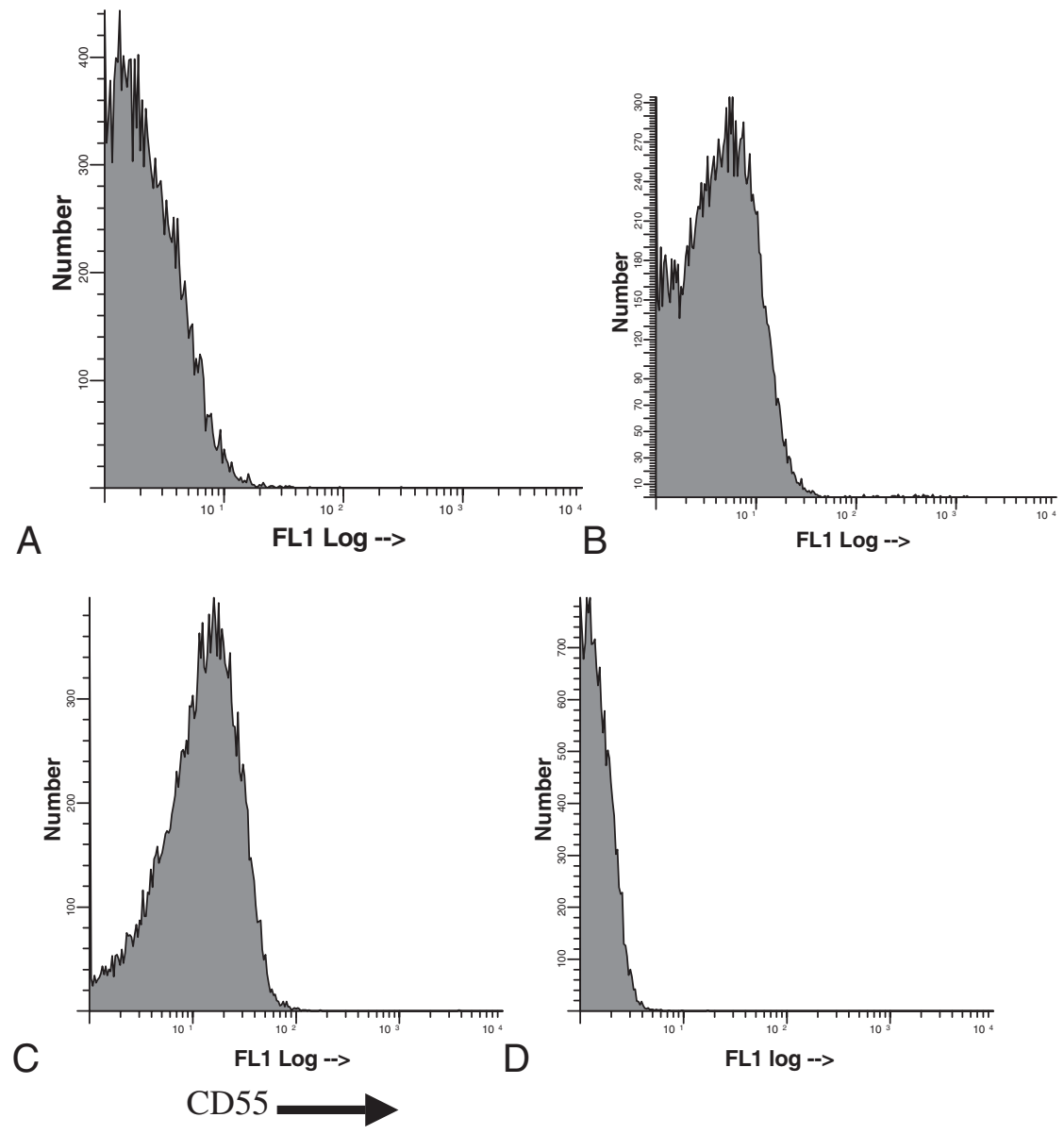

Fig. 1. Comparison of the expression of CD55 on the propositus' RBCs in August 2004 (A) and again in October 2004 (B). In August, most of his cells (98.4\%) had a complete absence of CD55. In October a significant proportion of his RBCs demonstrated increasing levels of CD55, and his circulating anti-IFC was virtually undetectable. (C) Positive control (RBCs donated by a healthy laboratory volunteer); (D) isotype 
reacted strongly at $4^{\circ} \mathrm{C}$ but there was also reactivity, albeit weaker, at IAT; however, reactivity was absent when saline was added in place of the AHG reagent. There is precedent for changes in the GI flora stimulating transient antiblood group antibodies: a 20-day old, K- neonate with sepsis caused by the uncommon E. coli variant O125:B15 produced anti-A and anti-K. Both antibodies were IgM and not of maternal origin. Cell-free isolates of these bacteria could inhibit the agglutination caused by these antibodies and thus bacteria were presumed to have been the sensitizing stimulus. When the infection resolved, the antibodies disappeared. ${ }^{27}$

Still, the stimulus for developing the current anti-IFC, as in the two previous reports of patients with transient Inab with anti-IFC remains unclear. ${ }^{21,22}$ The patient in the earlier report had been a blood donor for many years before developing this antibody, and no unexpected antibodies had been detected in his serum at the time of his donations..$^{21}$ The other patient with transient Inab phenotype had not been previously transfused, but had been pregnant (J. Poole, personal communication, June 2005). ${ }^{22}$ The current propositus was a very young male who had also never been transfused. Although we do not have a blood sample from before April 2004, the appearance and subsequent disappearance of the anti-IFC most likely correlates to the presence (and absence) of the corresponding antigen on the patient's RBCs. Anti-IFC is not invariably present in people with the Inab phenotype: a 70-year-old Italian-American individual who, along with his sister, inherited the Inab phenotype did not demonstrate antiIFC; given that both he and his sister demonstrated this rare phenotype, it is most likely inherited. Thus anti-IFC is not necessarily present in Inab individuals, even those with a genetic etiology to their phenotype. ${ }^{16}$ The appearance of anti-IFC was also noted in the original Inab propositus who presented with an anti-IFC; he had received transfusion of fresh-frozen plasma but apparently not RBCs. ${ }^{14}$

A common thread linking the first few reports of patients with the Inab phenotype was that these propositi had GI system abnormalities; the original propositus (Inab) had a protein-losing enteropathy, ${ }^{14}$ as did a 12 -yearold African American boy who almost certainly had the Inab phenotype.$^{18}$ Walthers and coworkers ${ }^{15}$ reported a third Inab phenotype patient who had Crohn's disease, whereas Daniels reported a Japanese woman with the Inab phenotype who suffered from blood capillary angioma of the small intestine. ${ }^{18}$ Although CD55 is known to be expressed on numerous epithelial surfaces including the gut, ${ }^{28}$ a causal association between its deficiency and anatomical lesions of the gut has not been established. DAF knock-out mice had heightened susceptibility to dextran sulfate sodium-induced colitis, suggesting its role in protecting the gut from insult. ${ }^{29}$ These similarities have to be interpreted in light of the six other Inab phenotype patients, including the two transient Inab patients, ${ }^{21,22}$ who did not demonstrate nor have a history of GI abnormalities. ${ }^{16,17,20}$

\section{ACKNOWLEDGMENTS}

MHY thanks Darrell Triulzi, MD, for thoughtful discussion and critical review of the manuscript.

\section{REFERENCES}

1. Daniels G. Human blood groups. 2nd ed. Oxford: Blackwell Science; 2002.

2. Reid ME, Powell V, Barnes J, et al. ZENA: a new high prevalence Cromer blood group antigen [abstract]. Transfusion 2004;44(Suppl):26A.

3. Storry JR, Sausais L, Hue-Roye K, et al. GUTI: a new antigen in the Cromer blood group system. Transfusion 2003;43:3404.

4. Banks J, Poole J, Ahrens N, et al. SERF. a new antigen in the Cromer blood group system. Transfus Med 2004;14:313-8.

5. Storry JR, Reid ME. The Cromer blood group system: a review. Immunohematology 2002;18:95-103.

6. Ivankovic Z, Golubic Cepulic B, Bekavac M, et al. CROV: a new high prevalence Cromer blood group antigen. Transfusion 2005;45(Suppl):122A.

7. Lublin DM. Review: Cromer and DAF: role in health and disease. Immunohematology 2005;21:39-47.

8. Rosse WF, Ware RE. The molecular basis of paroxysmal nocturnal hemoglobinuria. Blood 1995;86:3277-86.

9. Rosse WF, Nishimura J. Clinical manifestations of paroxysmal nocturnal hemoglobinuria. the present state and future problems. Int J Hematol 2003;77:113-20.

10. Levene C, Harel N, Lavie G, et al. A "new” phenotype confirming a relationship between $\mathrm{Cr}^{\mathrm{a}}$ and $\mathrm{Tc}^{\mathrm{a}}$. Transfusion 1984;24:13-5.

11. Lublin DM, Thompson ES, Green AM, Levene C, Telen MJ. Dr(a-) polymorphism of decay accelerating factor: biochemical, functional and molecular characterization and production of allele-specific transfectants. J Clin Invest 1991;87:1945-52.

12. Reid ME, Mallinson G, Sim RB, et al. Biochemical studies on the red blood cells from a patient with the Inab phenotype (decay accelerating factor deficiency). Blood 1991;78:3291-7.

13. Lublin DM, Mallinson G, Poole J, et al. Molecular basis of reduced or absent expression of decay-accelerating factor in Cromer blood group phenotypes. Blood 1994;84: 1276-82.

14. Daniels GL, Tohyama H, Uchikawa M. A possible null phenotype in the Cromer blood group complex. Transfusion 1982;22:362-3.

15. Walthers L, Salem M, Tessel J, et al. The Inab phenotype: another example found. Transfusion 1983;23(Suppl): 423. 
16. Lin RC, Herman J, Henry L, et al. A family showing inheritance of the Inab phenotype. Transfusion 1988;28: 427-9.

17. Wang L, Uchikawa M, Tsuneyama $\mathrm{H}$, et al. Molecular cloning and characterization of decay-accelerating factor deficiency in Cromer blood group Inab phenotype. Blood 1998;91:6804.

18. Daniels G, Green CA, Mallinson G, et al. Decay-accelerating factor (CD55) deficiency in Japanese. Transfus Med 1998;8: 141-7.

19. Uchikawa M, Tsuneyama H, Ogasawara K, et al. Another example of Inab phenotype in Japanese and production of human monoclonal anti-DAF. Vox Sang 2004;87(S3):41.

20. Hue-Roye K, Powell VI, Patel G, et al. Novel molecular basis of an Inab phenotype. Immunohematology 2005;21:53-5.

21. Matthes T, Tullen E, Poole J, et al. Acquired and transient RBC CD55 deficiency (Inab phenotype) anti-IFC. Transfusion 2002;42:1448-57.

22. Banks J, Poole J, Prowse C, et al. Transient loss of Cromer antigens and anti-IFC in a patient with chronic lymphatic leukemia. Vox Sang 2004;87(S3):37.

23. Judd WJ. Methods in immunohematology. 2nd ed. Durham (NC): Montgomery Scientific Publications; 1994.
24. Nowicki B, Moulds J, Hull R, et al. A hemagglutinin of uropathogenic Escherichia coli recognizes the Dr blood group antigen. Infect Immun 1988;56:1057-60.

25. Judd WJ, Cooling L. Novel application of the gel test: detection of microbial-induced hemagglutination. Vox Sang 2004;87(S3):76.

26. Telen MJ, Rao N, Udani M, et al. Molecular mapping of the Cromer blood group $\mathrm{Cr}^{\mathrm{a}}$ and $\mathrm{Tc}^{\mathrm{a}}$ epitopes of decay accelerating factor: toward the use of recombinant antigens in immunohematology. Blood 1994;84:3205-11.

27. Marsh WL, Nichols ME, Oyen R, et al. Naturally occurring anti-Kell stimulated by E. coli enterocolitis in a 20-day-old child. Transfusion 1978;18:149-54.

28. Medof ME, Walter EI, Rutgers JL, et al. Identification of the complement decay-accelerating factor (DAF) on epithelium and glandular cells and in body fluids. J Exp Med 1987; 165:848-64.

29. Lin F, Spencer D, Hatala DA, et al. Decay-accelerating factor deficiency increases susceptibility to dextran sulfate sodiuminduced colitis: role for complement in inflammatory bowel disease. J Immunol 2004;172:3836-41. 